\title{
Phase Separation in Disordered Exclusion Models
}

\author{
Joachim Krug \\ Fachbereich Physik, Universität GH Essen, D-45117 Essen, Germany \\ Received 15 October 1999
}

\begin{abstract}
The effect of quenched disorder in the one-dimensional asymmetric exclusion process is reviewed. Both particlewise and sitewise disorder generically induce phase separation in a range of densities. In the particlewise case the existence of stationary product measures in the homogeneous phase implies that the critical density can be computed exactly, while for sitewise disorder only bounds are available. The coarsening of phase-separated domains starting from a homogeneous initial condition is addressed using scaling arguments and extremal statistics considerations. Some of these results have been obtained previously in the context of directed polymers subject to columnar disorder.
\end{abstract}

\section{Introduction}

The one-dimensional asymmetric simple exclusion process (ASEP) was introduced by Spitzer in 1970 as an example of an interacting stochastic process [1, 2, 3]. In the probabilistic community it has been widely used for rigorous studies of the emergence of hydrodynamic behavior from stochastic microscopic dynamics [4, 5]. Already thirty years ago similar models were considered in the context of biopolymerization [6], while recent applications have focused on the problem of vehicular traffic flow [7]. The interest of statistical physicists has been further fueled by the discovery of boundary-induced phase transitions $[8,9,10]$ as well as the relations to interface growth and directed polymers in random media $[11,12]$. In short, the ASEP is a generic model of driven single file transport which combines utmost simplicity with a remarkable richness of behaviors.

Fig. 1 illustrates the model. Particles occupy the sites of a one-dimensional lattice subject to the simple exclusion rule (at most one particle per site). In an infinitesimal time interval $d t$ particle $i$ at site $x_{i}$ attempts a jump to the right (left) with probability $p d t$ ( $q d t$ ). The jump succeeds if the neighboring site is empty and is suppressed otherwise. In general the jump rates $p$ and $q$ may depend on both the particle label $i$ and the position $x$ on the lattice. In much of the paper I will restrict myself to the totally asymmetric case $q=0$.

In the present article I want to address the effects that quenched disorder in the jump rates has on the behavior of the ASEP. Disorder effects can be quite dramatic in one-dimensional single file systems, as is evidenced by the everyday experience with platoons and traffic jams caused by slow vehicles, accidents or road construction on highways $[13,14,15,16,17]$. Also in the context of driven transport on biomolecules a certain amount of disorder seems unavoidable [18].

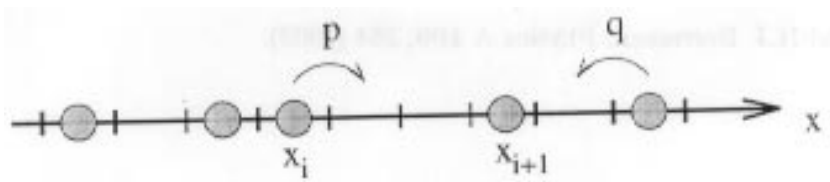

Figure 1. Illustration of the asymmetric simple exclusion process.

It is natural to distinguish between particlewise disorder with $p=p_{i}, q=q_{i}$ independent of $x$, and sitewise disorder with $p=p(x), q=q(x)$ independent of $i$. Both particlewise and sitewise disorder generically induce phase separation in the sense that, for global particle densities $\rho$ in a certain interval $\left[\rho_{c}^{-}, \rho_{c}^{+}\right]$, the system breaks up into regions of density $\rho_{c}^{-}$and $\rho_{c}^{+}$ separated by sharp density discontinuities ("shocks"). These shocks are typically associated with bottlenecks, i.e. slow particles or slow sites in the particlewise and sitewise cases, respectively. If the system is started from a homogeneous initial condition, the average size $\xi$ of phase separated regions grows as a power law

$$
\xi(t) \sim t^{1 / z},
$$

defining a dynamic exponent $z$; an example of the time evolution in the particlewise case is shown in Fig. 2. Two kinds of questions will therefore be asked in the following: First, how can the density interval $\left[\rho_{c}^{-}, \rho_{c}^{+}\right]$ of phase separation be determined? Second, what is the value of the dynamic exponent, and how does it depend on the distribution of the disordered jump rates? 


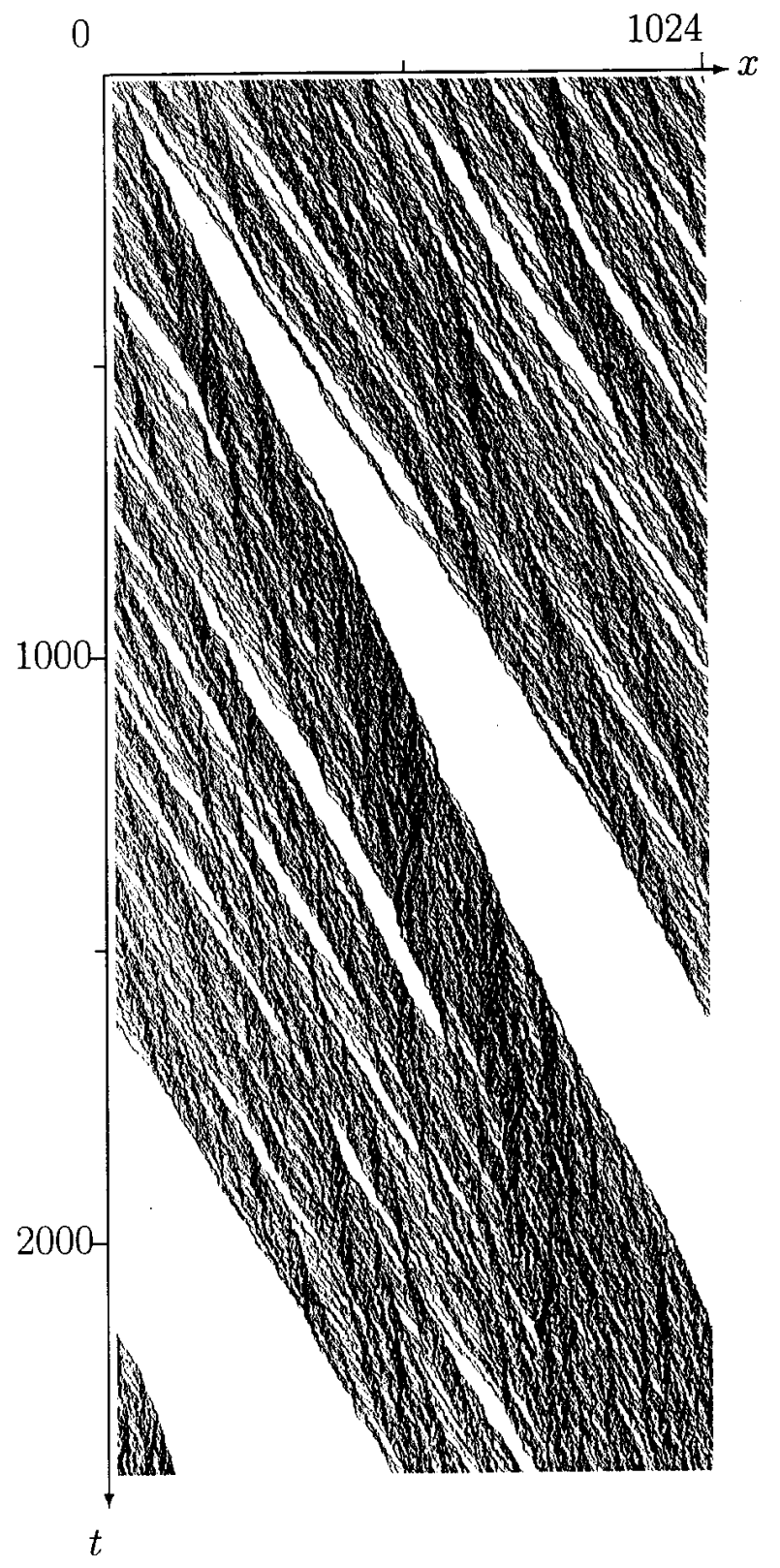

Figure 2. Space-time plot of trajectories of the ASEP with particlewise disorder. The figure shows 256 particles on a ring of 1024 sites. The initial distribution of particles was random with density $\rho=1 / 4<\rho_{c}=0.4$. Courtesy of $\mathrm{M}$. Gerwinski.

For particlewise disorder a number of exact analytic results are available [19, 20, 21, 22, 23] which have been reviewed elsewhere [15]. This case will therefore only be briefly summarized in Section II. The more difficult problem of sitewise disorder has been studied numerically by Tripathy and Barma [24] and others [16, 25], but little is known analytically. In Section III some progress in this direction will be reported. Specifically, I derive a rigorous bound on the critical densities based on the results for the particlewise case, and obtain predictions for the coarsening behavior for various types of disorder distributions. The relation to directed polymers in random media is briefly discussed in Section
III.4, and some conclusions and open questions are formulated in Section IV.

\section{Particlewise disorder}

\section{II.1 Steady state and critical density}

For particlewise disorder the configurations of the system are most naturally described in terms of the headways $u_{i}=x_{i+1}-x_{i}-1$ in front of the particles. The key simplifying feature is that different headways become statistically independent in the steady state, with a geometric distribution [19, 21]

$$
P_{i}(u)=\left(1-\alpha_{i}\right) \alpha_{i}^{u}
$$

for the headway in front of particle $i$. In the totally asymmetric case the parameters $\alpha_{i}$ are determined by the jump rates $p_{i}$ through the simple relation [20,21]

$$
\alpha_{i}=v / p_{i}
$$

where $v$ is the (common) mean speed of the particles in the steady state. Eq.(3) expresses the plausible fact that the headways in front of slow particles are larger than in front of fast ones. The geometric distribution (2) remains valid in the case of partial asymmetry, but then (3) is replaced by a more complicated relation $[19,21]$. The steady state distribution for the totally asymmetric model with parallel update has a similar form [22].

In the following we consider the totally asymmetric case and take the $p_{i}$ to be independent random variables with a probability density $f(p)$ supported on the interval $[c, 1]$, with a minimal speed $c$ bounded away from zero. Since particles cannot pass each other, it is clear that the steady state speed $v$ in an infinite system cannot exceed $c$. To compute it, one determines the mean headway in front of particle $i$ from (2) and performs the disorder average. In a system of density $\rho$ the resulting average headway must be $(1-\rho) / \rho$. This yields the implicit equation

$$
\rho=\left[1+v \int_{c}^{1} \frac{d p f(p)}{p-v}\right]^{-1}
$$

for the speed as a function of density. Two cases are to be distinguished. If the integral on the right hand side of (4) diverges in the limit $v \rightarrow c$, then $v(\rho)<c$ for all $\rho>0$. In this case the $\alpha_{i}$ in (2) are bounded away from unity for all $i$, the headway distributions are normalizable, and the system remains homogeneous. If, on the other hand, the integral remains finite in this limit, then the right hand side of (4) evaluated at $v=c$ defines a critical density $\rho_{c}$ such that $v(\rho) \equiv c$ in the entire interval $\left[0, \rho_{c}\right]$. For the slowest particles with $p_{i} \approx c$ this implies that the headway distributions (2) are no longer normalizable. Large gaps appear in front of these 
particles, and the faster particles form platoons behind them, a phenomenon familiar from vehicular traffic on country roads $[15,13,14]$. The system phase separates into regions of density $\rho_{c}^{-}=0$ (the gaps) and regions of density $\rho_{c}^{+}=\rho_{c}$ (the platoons).

It is evident from (4) that the condition for phase separation translates into a condition on the behavior of the disorder distribution $f(p)$ near $p=c$. Introducing an exponent $n$ through

$$
f(p) \sim(p-c)^{n}, \quad p \rightarrow c,
$$

phase separation occurs iff $n>0$. At the critical point $\rho=\rho_{c}$ the disorder averaged headway distribution has a power law tail $\sim u^{-(n+2)}$ [20]. Evans [21, 22] has emphasized the close analogy to Bose-Einstein condensation, where $f(p)$ plays the role of a density of states, and the slowest particle in the system corresponds to the quantum mechanical ground state.

\section{2 Coarsening behavior}

No exact results pertaining to the dynamics of phase separation are available, apart from the observation [23] that the existence of a well-defined hydrodynamic limit implies that inhomogeneities are restricted to scales smaller than $t$, and therefore

$$
\lim _{t \rightarrow \infty} \xi(t) / t=0
$$

Considerable evidence has however accumulated in favor of the idea [20] that the coarsening behavior for particlewise disorder can be described in terms of a simpler, deterministic model, in which particles move ballistically on the real line with fixed random speeds and coalesce upon overtaking. Such a model was first introduced by Newell [13], and later a detailed kinetic theory was worked out by Ben-Naim, Krapivsky and Redner [14].

Within the deterministic model, the dynamic exponent $z$ can be determined through a simple extremal statistics argument. The key idea is that the particles heading the platoons at time $t$ are those with the smallest speeds among of the order of $\xi(t)$ particles. Elementary probability theory suffices to show that, for a probability density behaving as $(5)$, these extremal speeds cluster in an interval of size $\xi^{-1 /(n+1)}$ above the minimal speed $c$. Therefore the speed difference $\Delta v$ between two platoons is of the order $\xi^{-1 /(n+1)}$, and the faster platoon will merge with the slower one on a time scale $t \sim \xi / \Delta v \sim \xi^{(n+2) /(n+1)}$. Inverting this relation one obtains the coarsening law (1) with

$$
z=\frac{n+2}{n+1} \text {. }
$$

Numerical results supporting (7) have been reported for models with parallel update [26], in simulations of jam dissolution [23] and in a simulation study of a system with open boundaries [27].

\section{Sitewise disorder}

\section{III.1 Disorder types}

We distinguish three cases which will turn out to represent different classes of coarsening behavior. For type I disorder the dynamics is totally asymmetric, $q(x) \equiv 0$, and the forward rates $p(x)$ are independent random variables in an interval $[c, 1]$, with a minimal rate $c>0$. The simplest (and typical) example is that of binary rates, with probability density

$$
f(p)=\phi \delta(p-c)+(1-\phi) \delta(p-1)
$$

where $\phi \in(0,1)$ denotes the fraction of slow sites. Type II disorder is similar to type I except that the support of the probability density $f(p)$ extends all the way to $p=0$, i.e. the minimal rate $c=0$. For type II disorder nontrivial dynamics occurs only for continuous $f(p)$. As in the models with particlewise disorder (eq.(5)), the important feature of $f(p)$ is the behavior near $p=0$, which can be characterized by an exponent $n$ through the relation

$$
f(p) \sim p^{n}, \quad p \rightarrow 0
$$

Finally, for type III disorder not only the strength, but also the direction of the bias is spatially random. A majority of sites has a bias to the right, say, with $p(x)>q(x)$, while a minority has $q(x)>p(x)$. If the one-dimensional lattice is viewed as a transport path in a higher-dimensional disordered structure, such as a percolation cluster, the stretches of minority sites can be interpreted as "backbends" where the path turns back against the direction of the driving field [28]. Compared to the strong disorder effects induced by the backbends, the randomness in the strength of the bias is irrelevant. Therefore a representative example of type III disorder is a model where the strength of the bias is constant, and only its direction varies. This corresponds to setting $q(x)=1-p(x)$ and choosing the $p(x)$ from a binary distribution which is symmetric around $p=1 / 2$,

$$
f(p)=(1-\phi) \delta(p-b)+\phi \delta(p-(1-b))
$$

Here $b \in(1 / 2,1)$ denotes the strength of the bias and $\phi \in(0,1 / 2)$ the fraction of minority sites.

It is easy to see that for type II and III disorder the stationary particle current vanishes in the infinite system limit, due to the existence of arbitrarily large stretches of arbitrarily small jump rates (for type II) or arbitrarily long backbends (for type III). As a consequence phase separation occurs at any density $\rho \in(0,1)$, i.e. $\rho_{c}^{-}=0$ and $\rho_{c}^{+}=1$. For type I disorder the existence of a nontrivial current function $J(\rho)>0$ describing the large scale dynamics of density profiles 
has been rigorously established, and it has been shown that $J(\rho)$ is convex in the sense that $J^{\prime \prime}(\rho) \leq 0$ [29]. However, in contrast to the models with particlewise disorder the stationary state is not known, and therefore an explicit computation of $J(\rho)$ is not possible. In the next section some bounds on $J(\rho)$ will be derived and used to bound the critical densities for type I disorder. The coarsening dynamics for all three cases will be addressed in Section III.3.

\section{III.2 Bounds on the critical density for type I disorder}

We first collect some obvious properties of $J(\rho)$. Due to particle-hole symmetry we have $J(\rho)=J(1-\rho)$. The current is bounded from below by the current $c \rho(1-\rho)$ of a pure system with all rates equal to the minimal rate $c$, and from above by the current $\rho(1-\rho)$ of the system with all rates equal to unity. A more precise upper bound is obtained by observing that in the infinite system there are arbitrarily large stretches with rates arbitrarily close to $c$. The maximum current that can be driven through such a stretch is $c / 4$, the maximum value of $c \rho(1-\rho)$. We conclude that

$$
c \rho(1-\rho) \leq J(\rho) \leq \min [c / 4, \rho(1-\rho)] .
$$

Numerical simulations of site-disordered exclusion models [24, 16, 25] and related growth models [30, 31] indicate that the upper bound $c / 4$ is attained in a finite density interval around $\rho=1 / 2$, which coincides with the phase separation interval $\left[\rho_{c}^{-}, \rho_{c}^{+}\right]$; by particle-hole symmetry $\rho_{c}^{-}=1-\rho_{c}^{+} \equiv \rho_{c}$. In the following our strategy will be to derive optimal lower and upper bounds $J_{<}(\rho)$ and $J_{>}(\rho)$ on the stationary current, which are then translated into lower and upper bounds $\rho_{c}^{<}, \rho_{c}^{>}$on $\rho_{c}$ through the relation

$$
J_{>}\left(\rho_{c}^{<}\right)=J_{<}\left(\rho_{c}^{>}\right)=c / 4 .
$$

The lower current bound in (11) does not give rise to any nontrivial density bound, while the upper bound $\rho(1-\rho)$ yields

$$
\rho_{c} \geq(1-\sqrt{1-c}) / 2 .
$$

For the case of binary disorder (eq.(8)) an improved lower bound on the current was derived by Tripathy and Barma [24] by considering a finite ring of $L$ sites, $N=\rho L$ particles and $N_{s}=\phi L$ slow sites. They start from the observation that the maximum current that can be driven through a stretch of slow sites is a decreasing function of the length of the stretch (we will return to this point below in Section III.3). It is therefore plausible (though not rigorously established) that for given $L, N$ and $N_{s}$ the stationary current will be minimal in the fully segregated limit where all slow sites form a single large stretch. For $L \rightarrow \infty$ the fully segregated system can be treated as two connected homogeneous systems with different densities, which are fixed through the constraints of equal currents and total particle number. This yields the upper density bound

$$
\rho_{c} \leq(1-(1-\phi) \sqrt{1-c}) / 2
$$

In the dilute limit $\phi \rightarrow 0$ the bounds (13) and (14) coincide, and give $\rho_{c}=(1-\sqrt{1-c}) / 2$ exactly. It should however be noted that this limit does not correspond to the case of a single defect site, since the maximal current that can be driven through a single defect is larger than $c / 4$ [32] (see also Section III.3).

The lower bound (13) can be improved by comparing the disordered exclusion model to a zero range process (ZRP) with the same set of jump rates $\{p(x)\}$. In the ZRP an arbitrary number of particles is allowed on any site $[1,15]$, and therefore any attempted jump succeeds. As a consequence the stationary state of the ZRP is a product measure, with the occupation numbers at different sites being independent, for any choice of jump rates depending on the position $x$ and on the number of particles at the site $[19,33]$. Here we consider the case where the rate at which a particle is transferred from site $x$ to $x+1$ is equal to $p(x)$ independent of the number of particles at $x$, provided the latter is not zero. It is then obvious (and can be proved through waiting time considerations) that the particle current $J_{\mathrm{ZRP}}(\rho)$ of the ZRP provides an upper bound to the current $J(\rho)$ of the ASEP.

In fact the disordered ZRP is equivalent to the ASEP with particlewise disorder, with the ZRP occupation numbers representing the headways in the ASEP $[19,15]$. The ZRP current is equal to the particle speed $v$ of the ASEP, which is given by (4) for any disorder distribution $f(p)$. The ZRP density is equal to the mean headway of the ASEP, and is therefore related to the ASEP density through $\rho_{\mathrm{ZRP}}=1 / \rho_{\mathrm{ASEP}}-1$. Evaluating the integral in (4) for the binary distribution (8) yields

$$
\rho_{\mathrm{ZRP}}=J_{\mathrm{ZRP}}\left(\frac{\phi}{c-J_{\mathrm{ZRP}}}+\frac{1-\phi}{1-J_{\mathrm{ZRP}}}\right)
$$

and setting $J_{>}=J_{\mathrm{ZRP}}$ in (12) we obtain the density bound

$$
\rho_{c} \geq \frac{\phi}{3}+\frac{c(1-\phi)}{4-c}
$$

which improves (13) for small $c$. In particular, for $c \rightarrow 0$ we have $\rho_{c} \geq \phi / 3$, which proves, remarkably, that the homogeneous phase $\rho<\rho_{c}$ persists even when the slow sites become complete blockages. In Fig. 3 the bounds (13), (14) and (16) are compared to numerical data. 


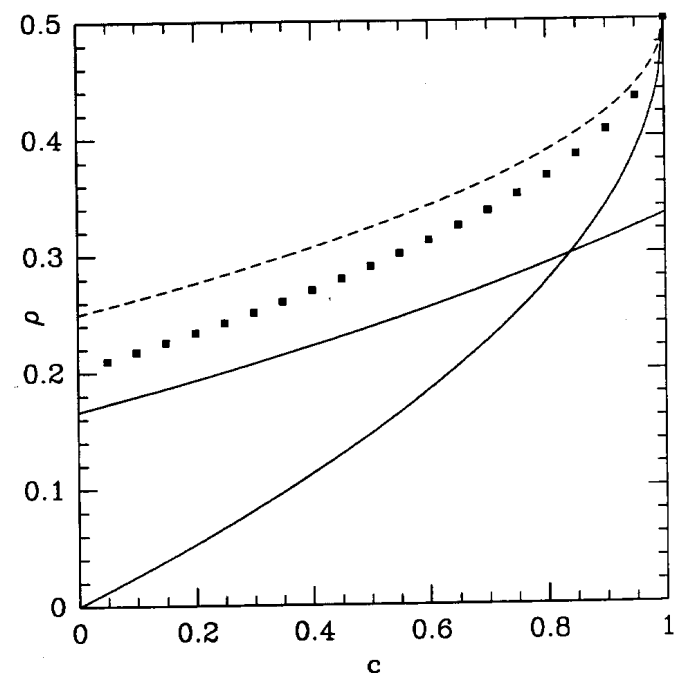

Figure 3. Bounds on the critical density for sitewise disorder with the binary distribution (8), $\phi=1 / 2$. The dashed line is the upper bound (14), the two full curves show the lower bounds (13) and (16), and the full squares are simulation data obtained by G. Tripathy.

\section{III.3 Coarsening behavior}

At least for type I disorder the existence of a hydrodynamic limit [29] implies that the relation (6) carries over to the sitewise case. To obtain a finer estimate of the coarsening scale $\xi(t)$ we rely on extremal statistics arguments similar to those used in Section II.2. A schematic phase separated density profile is shown in Fig. 4. Two "antischocks" at positions $x_{1}$ and $x_{2}$, where the density jumps from $\rho_{c}^{+}=1-\rho_{c}$ to $\rho_{c}^{-}=\rho_{c}$, mark bottleneck regions of particularly slow rates, which support maximum currents $j_{1}$ and $j_{2}$. If the bottleneck in the downstream direction is slightly more restrictive, in the sense that $\Delta j=j_{1}-j_{2}>0$, then the low density region between the bottlenecks will slowly fill in and disappear at a time

$$
t \approx\left(1-2 \rho_{c}\right) \xi / \Delta j
$$

If the statistics of extremal bottlenecks is known, the typical current difference $\Delta j$ can be estimated as a function of $\xi$ and (17) yields a prediction for the coarsening law $\xi(t)$. In the following this will be carried out for the different disorder types.

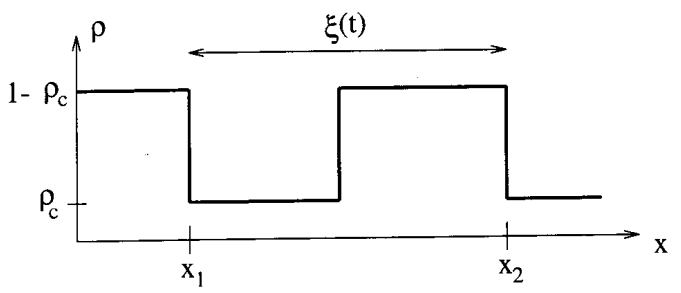

Figure 4. Schematic of two bottlenecks at positions $x_{1}$ and $x_{2}$.

\section{III.3.1 Type I disorder}

Consider first the conceptually simplest case of the binary disorder distribution (8). We expect long stretches of slow sites to constitute the most restrictive bottlenecks. For a quantitative analysis we would require the maximum current $j_{\max }(c, \ell)$ which can be driven through a stretch of $\ell$ slow sites with jump rates $c$ embedded in an infinite system of sites with jump rates 1 . Already for $\ell=1$ the computation of $j_{\max }(c, \ell)$ is a difficult unsolved problem [32]. However for large $\ell$ we can make progress by replacing the stretch by a finite system of $\ell$ sites with uniform jump rates $c$ and periodic or open boundary conditions, for which the maximum current is known [9, 10]. For both kinds of boundary conditions the current approaches the $\ell \rightarrow \infty$ limit $c / 4$ from above, with a leading correction [34] proportional to $1 / \ell$. Thus we expect, for large $\ell$,

$$
j_{\max }(c, \ell) \approx(c / 4)(1+a / \ell)+\mathcal{O}\left(1 / \ell^{2}\right),
$$

where $a$ is a positive constant of order unity.

Since the probability distribution of the lengths of slow stretches is

$$
P(\ell)=(1-\phi) \phi^{\ell},
$$

the longest stretch in a region of size $\xi$ is of the order of

$$
\ell_{\max } \approx \frac{\ln \xi}{\ln (1 / \phi)}
$$

Note that $\ell_{\max } \ll \xi$, which is consistent with the assumption of well-localized bottlenecks inherent in Figure 4. Using (18) we see that the currents supported by the longest stretches exceed $c / 4$ by an amount of the order of $c / \ell_{\max }$, and therefore

$$
\Delta j \approx \frac{c \ln (1 / \phi)}{\ln \xi}
$$

Inserting this into (17) the leading order coarsening law is obtained as

$$
\xi(t) \sim \frac{t / t_{0}}{\ln \left(t / t_{0}\right)}
$$

with a characteristic time scale $t_{0} \sim\left(1-2 \rho_{c}\right) / c \ln (1 / \phi)$. This argument was formulated earlier in the context of phase-disordered growth models, where also numerical evidence in favor of the coarsening law (22) was presented [30, 31].

For continuous disorder distributions $f(p)$ the identification of the relevant bottlenecks is a little more subtle. Consider a region of size $\ell$ where all rates satisfy $p(x) \leq c+\epsilon$. The maximum current through such a region can then be estimated as

$$
j_{\max } \approx \frac{c+\epsilon}{4}\left(1+\frac{a}{\ell}\right) \approx c / 4+\epsilon / 4+c a / 4 \ell \equiv c / 4+j .
$$


If $f(p)$ behaves as in (5) for $p \rightarrow c$ the probability of the region is of the order of $\epsilon^{(n+1) \ell}$. The probability distribution of $j$ can then be written as

$$
\begin{gathered}
P(j) \sim \int d \ell \int d \epsilon \epsilon^{(n+1) \ell} \delta(j-(\epsilon+c a / \ell) / 4) \sim \\
\int d \epsilon \exp [-(n+1) c a \ln (1 / \epsilon) /(4 j-\epsilon)] .
\end{gathered}
$$

Evaluating the last integral at the saddle point yields

$$
P(j) \sim \exp \left[-(n+1) c a \ln ^{2}(1 / j) / j\right]
$$

The current scale $\Delta j$ of the most restrictive among $\xi$ bottlenecks is obtained by setting $P(\Delta j) \sim 1 / \xi$, which gives

$$
\Delta j \sim \frac{[\ln (\ln \xi)]^{2}}{\ln \xi},
$$

and the corresponding coarsening law reads, to leading order in $t$,

$$
\xi(t) \sim \frac{t[\ln (\ln t)]^{2}}{\ln t}
$$

which, for most purposes, is indistinguishable from (22).

\section{III.3.2 Type II and III disorder}

For type II disorder with a continuous probability distribution $f(p)$, characterized by $(9)$, the expression (23) for the maximum current supported by a slow stretch of length $\ell$ applies with $c=0$. The distribution of $j_{\max }$ then becomes

$$
\begin{gathered}
P\left(j_{\max }\right) \sim \int d \ell \int d \epsilon \epsilon^{(n+1) \ell} \delta\left(j_{\max }-\epsilon(1+a / \ell) / 4\right) \sim \\
\int d \ell \exp \left[-(n+1) \ell \ln \left((1+a / \ell) / 4 j_{\max }\right)\right] .
\end{gathered}
$$

Now the maximum of the exponent evidently occurs at $\ell=1$, i.e. the dominant bottlenecks are individual slow sites. The distribution of the currents supported by the bottlenecks is then simply given by the jump rate distribution $f(p)$ itself, and the situation reduces to that analyzed in the case of particlewise disorder, Section II.2. In particular, the coarsening exponent $z$ for type II sitewise disorder is also given by $(7)$.

For type III disorder with distribution (10) the dominant bottlenecks are long backbends, i.e. stretches of minority sites at which the local bias is directed against the mean flow direction. The maximum current that can be driven through a backbend of length $\ell$ is exponentially small in $\ell$, and is given by [24, 28]

$$
j_{\max }(\ell) \sim \exp [-(1 / 2) \ell \ln (b /(1-b))] .
$$

Combining this with the probability distribution (19) of backbend lengths it follows that $j_{\max }$ is distributed according to a power law,

$$
P\left(j_{\max }\right) \sim\left(j_{\max }\right)^{2 \theta^{-1}-1}
$$

where

$$
\theta=\frac{\ln [b /(1-b)]}{\ln [1 / \phi]}
$$

Since the largest backbend in a region of size $\xi$ is of length $\ell \sim \ln \xi \ll \xi$, we can employ a coarse grained picture in which the backbends are shrunk to individual sites with a jump rate distribution given by $(30)$, thus effectively reducing the problem to type II disorder with the exponent $n$ in (9) given by $n=2 / \theta-1$. The coarsening exponent for the disorder distribution (10) is then obtained from (7) as

$$
z=1+\theta / 2
$$

\section{III.4 Relation to directed polymers}

Using the waiting time approach [35] the site disordered ASEP can be mapped to a zero temperature directed polymer (DP) with point and columnar disorder [36]. In that context the coarsening law $\xi(t)$ describes the disorder-induced transverse wandering of the polymer, which can be estimated using variable range hopping arguments [36] and the analogy to Lifshitz tails for one-dimensional disordered Schrödinger operators [37].

To see that the results derived for the DP are consistent with those obtained above, it is important the recall [35] that the waiting time mapping transforms the time $t$ of the ASEP into the energy of DP. For type I disorder the transverse wandering $\delta x$ of the DP was found to increase with its length $L$ as [36]

$$
\delta x \sim L /(\ln L)^{2},
$$

while the ground state energy behaves as $E \sim L / \ln L$ to leading order. Combining the two results and identifying $E \sim t$ the coarsening law (22) follows.

For type II disorder the power law (9) of the probability distribution at small $p$ translates into a power law tail

$$
P(\tau) \sim \tau^{-(2+n)}, \quad \tau \rightarrow \infty
$$

in the distribution of waiting times or energies $\tau=1 / p$. Directed polymers in the presence of columnar disorder with a power law distribution were considered in Ref.[37], where it was shown that the wandering is typically ballistic, $\delta x \sim L$, while the ground state energy scales with length as

$$
E \sim L^{(n+2) /(n+1)}
$$

in agreement with (7). It is worthwhile to point out that in the DP context the scaling laws (33) and (35) were also confirmed numerically $[36,37]$. 


\section{Summary and open ques- tions}

In this paper I have described some recent progress in our understanding of disorder effects in asymmetric simple exclusion models. A common feature of both particlewise and sitewise disordered systems is the appearance of phase separation in an interval of densities, which is macroscopically characterized by a linear portion in the current-density relation $J(\rho)$; in the particlewise case $J(\rho)=c \rho$ for $\rho<\rho_{c}$, while in the sitewise case $J(\rho) \equiv c / 4$ for $\rho_{c} \leq \rho \leq 1-\rho_{c}$. An interesting open question concerns the connection between phase separation and linearity of $J(\rho)$, which is reminiscent of the role that the convexity of thermodynamic potentials plays for the stability of equilibrium systems. While it is obvious that phase separation implies a linear segment in $J(\rho)$, the converse statement has, to my knowledge, not been established. To prove that it is false, it would be sufficient to find a (noisy!) exclusion type model with a homogeneous stationary state and a linear current-density relation (deterministic systems with linear $J(\rho)$ are well known $[7,38]$ ).

The dynamics of phase separation has been explored in the framework of scaling arguments, which can be formulated in a similar way both for particlewise and sitewise disorder. In the particlewise case the relevant bottlenecks which determine the positions of domain boundaries are always individual slow particles, while in the sitewise case with type I and III disorder the bottlenecks are formed collectively by many defects. For type I disorder this implies a certain universality of the coarsening law, in the sense that the exponent $z$ in (1) is $z=1$ independent of the underlying disorder distribution; the additional logarithmic corrections in $(22,27)$ ensure the consistency with the rigorous result (6). This is somewhat analogous to the case of finite temperature directed polymers with columnar defects, where universal scaling laws arise from the thermal averaging over large spatial regions [37].

A numerical confirmation of the predictions for the coarsening dynamics in the case of sitewise disorder would be most welcome. For type II disorder this should be relatively straightforward, however in the cases of type I and III disorder the behavior is dominated by exponentially rare regions, which may make it hard to reach asymptopia.

Acknowledgements The results on particlewise disorder were obtained in collaboration with Pablo Ferrari and Timo Seppäläinen, while the material on sitewise disorder is joint work with Mustansir Barma and Goutam Tripathy. Support by DAAD within the PROBRAL programme and by DFG within SFB 237 is gratefully acknowledged.

\section{References}

[1] F. Spitzer, Adv. in Math. 5, 256 (1970).

[2] T.M. Liggett, Interacting Particle Systems (Springer, Berlin, 1985).

[3] T. Liggett, Stochastic Interacting Systems: Contact, Voter and Exclusion Processes (Springer, Berlin/Heidelberg, 1999).

[4] H. Spohn, Large Scale Dynamics of Interacting Particles (Springer, Berlin/Heidelberg, 1991).

[5] C. Kipnis and C. Landim, Scaling Limits of Interacting Particle Systems (Springer, Berlin/Heidelberg, 1999).

[6] G. Schütz, Int. J. Mod. Phys. B 11, 197 (1997), and references therein.

[7] K. Nagel, Phys. Rev. E 53, 4655 (1996).

[8] J. Krug, Phys. Rev. Lett. 67, 1882 (1991).

[9] G. Schütz and E. Domany, J. Stat. Phys. 72, 277 (1993).

[10] B. Derrida, M. R. Evans, V. Hakim, and V. Pasquier, J. Phys. A 26, 1493 (1993).

[11] J. Krug and H. Spohn, in Solids Far From Equilibrium, edited by C. Godrèche (Cambridge University Press, Cambridge, 1991), p. 479.

[12] T. Halpin-Healy and Y.C. Zhang, Phys. Rep. 254, 215 (1995).

[13] G.F. Newell, Opns. Res. 7, 589 (1959).

[14] E. Ben-Naim, P. Krapivsky, S. Redner, Phys. Rev. E 50, 822 (1994).

[15] J. Krug, in "Traffic and Granular Flow '97'", edited by M. Schreckenberg and D. E. Wolf (Springer, Singapore, 1998), p. 285.

[16] Z. Csahok and T. Vicsek, J. Phys. A 27, L591 (1994).

[17] W. Knospe, L. Santen, A. Schadschneider and M. Schreckenberg, in "Traffic and Granular Flow ' 97 '", ed. by M. Schreckenberg and D. E. Wolf (Springer, Singapore, 1998), p. 349.

[18] T. Harms and R. Lipowsky, Phys. Rev. Lett. 79, 2895 (1997).

[19] I. Benjamini, P.A. Ferrari and C. Landim, Stoch. Proc. Appl. 61, 181 (1996).

[20] J. Krug and P.A. Ferrari, J. Phys. A 29, L465 (1996).

[21] M.R. Evans, Europhys. Lett. 36, 13 (1996).

[22] M.R. Evans, J. Phys. A 30, 5669 (1997).

[23] T. Seppäläinen and J. Krug, J. Stat. Phys. 95, 525 (1999).

[24] G. Tripathy and M. Barma, Phys. Rev. E 58, 1911 (1998).

[25] M. Bengrine, A. Benyoussef, H. Ez-Zahraouy and F. Mhirech, Phys. Lett. A 253, 135 (1999). 
[26] D. V. Ktitarev, D. Chowdhury and D. E. Wolf, J. Phys. A 30, L221 (1997).

[27] M. Bengrine, A. Benyoussef, H. Ez-Zahraouy, J. Krug, M. Loulidi and F. Mhirech, J. Phys. A 32, 2527 (1999).

[28] R. Ramaswamy and M. Barma, J. Phys. A 20, 2973 (1987).

[29] T. Seppäläinen, Ann. Probab. 27, 361 (1999).

[30] J. Krug, in "Surface Disordering: Growth, Roughening and Phase Transitions", edited by R. Jullien, J. Kertész, P. Meakin and D.E. Wolf (Nova Science, 1992), p. 177 .

[31] J. Krug, Phys. Rev. Lett. 75, 1795 (1995).
[32] S. A. Janowsky and J. L. Lebowitz, Phys. Rev. A 45, 618 (1992); S. A. Janowsky and J. L. Lebowitz, J. Stat. Phys. 77, 35 (1994).

[33] E. D. Andjel, Ann. Probab. 10, 525 (1982).

[34] J. Krug and P. Meakin, J. Phys. A 23, L987 (1990).

[35] J. Krug and L.-H. Tang, Phys. Rev. E 50, 104 (1994).

[36] I. Arsenin, T. Halpin-Healy and J. Krug, Phys. Rev. E 49, R3561 (1994).

[37] J. Krug and T. Halpin-Healy, J. Physique I France 3, 2179 (1993).

[38] K. Nagel and H.J. Herrmann, Physica A 199, 254 (1993). 